\title{
Video Article \\ Visualization of Intensity Levels to Reduce the Gap Between Self-Reported and Directly Measured Physical Activity
}

\author{
Lisa Voigt $^{1,4}$, Antje Ullrich ${ }^{1,4}$, Ulrike Siewert-Markus ${ }^{1,2,4}$, Marcus Dörr ${ }^{3,4}$, Ulrich John ${ }^{1,4}$, Sabina Ulbricht ${ }^{1,4}$ \\ ${ }^{1}$ Institute of Social Medicine and Prevention, University Medicine Greifswald \\ ${ }^{2}$ Institute for Medical Psychology, University Medicine Greifswald \\ ${ }^{3}$ Department of Internal Medicine B, University Medicine Greifswald \\ ${ }^{4}$ partner site Greifswald, DZHK (German Centre for Cardiovascular Research)
}

Correspondence to: Lisa Voigt at lisa.voigt@uni-greifswald.de

URL: https://www.jove.com/video/58997

DOI: doi:10.3791/58997

Keywords: Behavior, Issue 145, self-report, accelerometry, physical activity, validity, intensity levels, moderate-to-vigorous, physical activity questionnaire, video, bias, treadmill, exercise, behavior

Date Published: $3 / 7 / 2019$

Citation: Voigt, L., Ullrich, A., Siewert-Markus, U., Dörr, M., John, U., Ulbricht, S. Visualization of Intensity Levels to Reduce the Gap Between SelfReported and Directly Measured Physical Activity. J. Vis. Exp. (145), e58997, doi:10.3791/58997 (2019).

\section{Abstract}

Physical activity (PA) assessment needs tools that are inexpensive and easy to administer. Common questionnaires inquire time spent in light, moderate, and vigorous PA. However, inaccuracies may occur due to individually different understanding of PA intensity levels. Alternatively used direct measures (e.g., accelerometers) are susceptible to reactivity bias and may lack the ability to capture certain activities. Compared to accelerometer measurement, respondents report more time spent in higher-intensity PA. A video that visualizes PA intensity levels might help to overcome this problem. This report describes the design of a randomized controlled trial as a methodology to investigate the effect of a video on the difference between self-reported and directly measured PA. It is hypothesized that the video reduces the mean difference between the two measures. Individuals from the general population are recruited. Hip-worn accelerometers are used to collect directly measured PA data on seven consecutive days. Afterwards, participants are randomly allocated to the experimental and the control group. The experimental group receives a video demonstration on PA intensity levels and subsequent PA assessment via self-administered computer-assisted questionnaire. The control group receives PA assessment only. Thereafter, the data are processed to compare the difference between self-reported and accelerometer-based moderate-to-vigorous physical activity (MVPA) between the study groups using a two-sample t-test. This methodology is appropriate for investigating the effect of any existing or self-produced video on the difference between the two measurement methods. It can be used not only for persons from the general population, but for a variety of other populations and contexts as accurate measures are needed to evaluate PA levels.

\section{Video Link}

The video component of this article can be found at https://www.jove.com/video/58997/

\section{Introduction}

Assessment of physical activity (PA) is commonly done by questionnaires because they are inexpensive and easy to administer. As positive associations between amounts of higher-intensity PA and cardiovascular health are well established $d^{1,2,3}$, many questionnaires inquire frequency and time spent in light, moderate, and vigorous PA presenting examples of respective activities ${ }^{4,5,6,7,8}$. However, they may be flawed by inaccuracy due to individually different understanding of PA intensity levels ${ }^{9}$. Further, specific activity examples may not hold true for individuals with different physical constitutions. For example, overweight or obese persons may feel more exerted than persons with normal weight when performing the exact same activity. Direct measures on the other hand (e.g., accelerometry) require considerable amounts of time and costs and possess limited validity due to reactivity bias ${ }^{10,11}$, sample selection bias ${ }^{12}$, and the lack of ability to accurately capture certain activities ${ }^{13}$. A broad range of studies showed only low to moderate agreements between self-reported and accelerometer-based PA ${ }^{14,15,16}$. Most findings indicate that respondents report more time spent in higher-intensity PA compared to directly measured data. Throughout the manuscript, the term "gap" is used to designate this lack of agreement between accelerometry and self-reported PA.

A video as part of a computer-assisted self-completed questionnaire might help to reconcile the two measures by increasing the accuracy of self-reports. A video demonstration provides an opportunity to show different intensity levels of PA that are hard to explain by written text only. Respondents receive a visual reference they may compare their performance levels with and thus, misclassification of light, moderate, and vigorous PA may be reduced. Up to now, videos to support assessments are available in the context of mobility and physical functioning validated for older adults ${ }^{17,18,19}$. To our knowledge, there are no video-supported assessments that provide a reference for light, moderate, and vigorous PA.

We developed a 3-minute video showing a middle-aged man on a treadmill in a fitness center who describes the terms light, moderate, and vigorous PA and simultaneously visualizes symptoms related to these intensity levels. The methodology described here is a randomized 
controlled trial to test the effect of the video demonstration on the gap between self-reported and accelerometer-based moderate-to-vigorous physical activity (MVPA). In addition, standardized assessment of somatometry (height, body weight, and waist and hip circumference) is conducted to investigate whether effects differ according to participants' physical constitution.

The methodology is appropriate to test the effect of any video demonstration that is meant to support computer-assisted PA questionnaire assessment with the aim of reducing the gap between self-reported and directly measured PA. The methodology can be used in various populations and contexts as accurate measures are needed to evaluate current and changing PA levels, efficacy of PA interventions, and associations between PA and health outcomes.

\section{Protocol}

This protocol was approved by the ethics committee of the University Medicine Greifswald (number BB 076/18; June 2018).

\section{Video construction and experimental design}

1. Select a publicly available or self-produced video based on the specific experimental question. The video should explain the terms used in the self-report questionnaire to support participants' understanding. The video used here contains explaining and visualizing symptoms as well as naming examples of light, moderate, and vigorous PA.

1. In the video, have a person on a treadmill in a fitness center give a general introduction to the different intensity levels of PA.

2. Have the person explain differences in heart rate, breathing frequency, and capability to talk normally in accordance with the intensity levels. Have the person simultaneously demonstrate those symptoms while walking/running on a treadmill at the according pace.

3. Have the person give examples of daily-life activities and emphasize individual differences in the evaluation of PA intensity levels. NOTE: The video used here was produced in German based on a video clip from the Centers for Disease Control and Prevention $(C D C)^{20}$. If participants are native English speakers, the original video may be used with emphasis on minutes 1:46 to 3:25. The person in the present video is an approximately fifty-year-old, normal-weight, white male in good physical shape. See Figure 1 for a visual depiction of video structure and contents.

2. Integrate the video into a self-administered tablet-computer survey to be presented directly before the PA questionnaire and make sure participants cannot skip the video. Randomize presentation of the video 1:1.

1. Integrate questions on sociodemographic and health related variables into the survey as desired for description of sample characteristics.

2. In the present study, self-reported PA is assessed using a modified version of the International Physical Activity Questionnaire Short Form (IPAQ-SF) ${ }^{4}$, German version ${ }^{21}$, addressing the last seven days. Two items each address number of days and respective time spent in moderate and vigorous PA. The original items on walking are replaced with questions on light PA as walking may be performed on different intensity levels ${ }^{22}$ and walking is not equivalent to light PA measured by accelerometry. Sociodemographic and health variables included in the survey are sex, age, school education, employment, current living together with a partner, current smoking, and self-rated general health. 

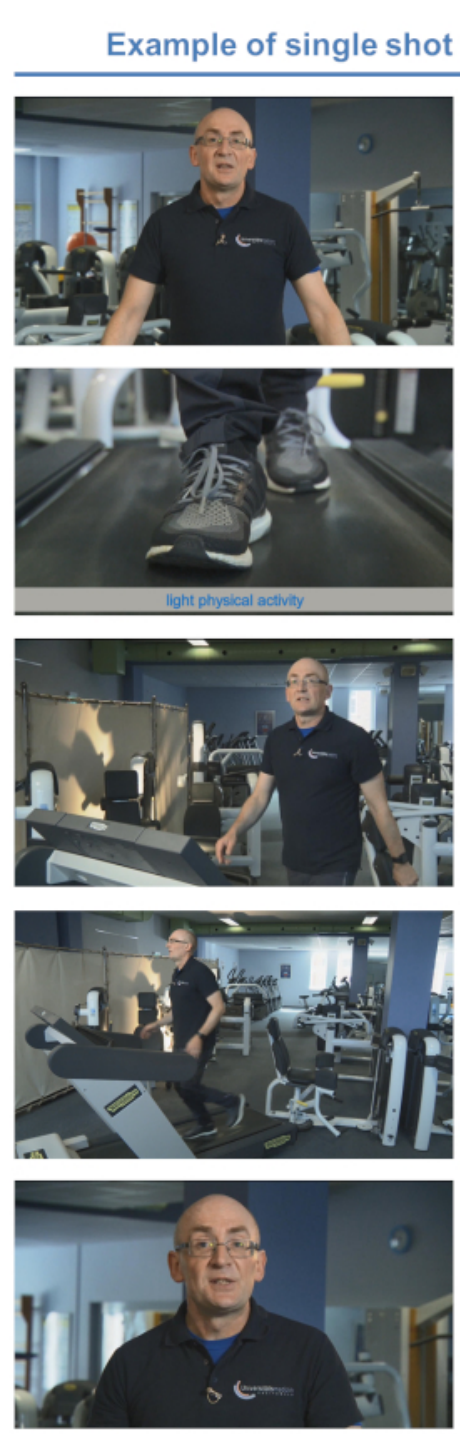

\section{Summary of content}

\author{
0:00 Introduction \\ - Action of the person: standing \\ - Explaining the purpose of the \\ demonstration and the definition \\ of "intensity"
}

Light physical activity

- Action of the person: walking

- Normal heart rate and breathing, normal capability to talk

- Examples: light housework, shopping

\section{$1: 13$ \\ Moderate physical activity}

- Action of the person: brisk walking

- Slightly increased heart rate and breathing, slightly lowered capability to talk

- Examples: brisk cycling, lawn mowing

1:51 Vigorous physical activity

- Action of the person: running

- Explicitly increased heart rate and breathing, explicitly lowered capability to talk

- Examples: running, playing soccer

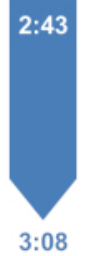

\section{Ending}

- Action of the person: standing

- Summary with emphasis on individually different intensity perceptions of activity examples

Figure 1: Schematic structure of the video demonstration of different physical activity intensity levels. The main scenes of the video with according single shots, lengths, and summary of contents are depicted. The video was based on a video clip provided by the CDC ${ }^{20}$. Please click here to view a larger version of this figure.

\section{Power calculation}

1. Conduct a power analysis using respective software in order to define the sample size necessary to obtain statistically conclusive results. Include an interim analysis to verify underlying assumptions and early stopping of the study.

1. Choose a statistical test appropriate for the research question.

2. Based on the literature, set the assumed mean difference between questionnaire and accelerometer data in the control group, that is, the divergence between self-reported and directly measured PA without presentation of the video.

3. Set the assumed mean difference between questionnaire and accelerometer data in the experimental group, that is, the divergence between self-reported and directly measured PA with inclusion of the video demonstration.

4. Set the assumed standard deviation (SD) for both groups.

5. Choose power and alpha-level as desired.

2. Based on the literature and considering the specific study design, decide on an assumed drop-out rate to retrieve the final number of participants to be recruited.

3. The power analysis of the present study is based on a two-sample t-test assuming equal variance. Based on a comparable sample ${ }^{10}$, the assumed mean difference between questionnaire and accelerometer data in the control group is 90 min per day of MVPA. The assumed mean difference in the experimental group is $60 \mathrm{~min}$ per day ( $S D$ in both groups $=100 \mathrm{~min}$ per day). As it is hypothesized that the integration of the video reduces the gap between the two measures, a one-sided significance level of $p=.05$ is chosen (power $=.80$ ). Results of power calculation including interim analysis revealed that a total number of 314 participants is needed for demonstrating the experimental effect. Assuming a drop-out rate of about $10 \%$, it is planned to recruit 350 participants (Figure 2). 


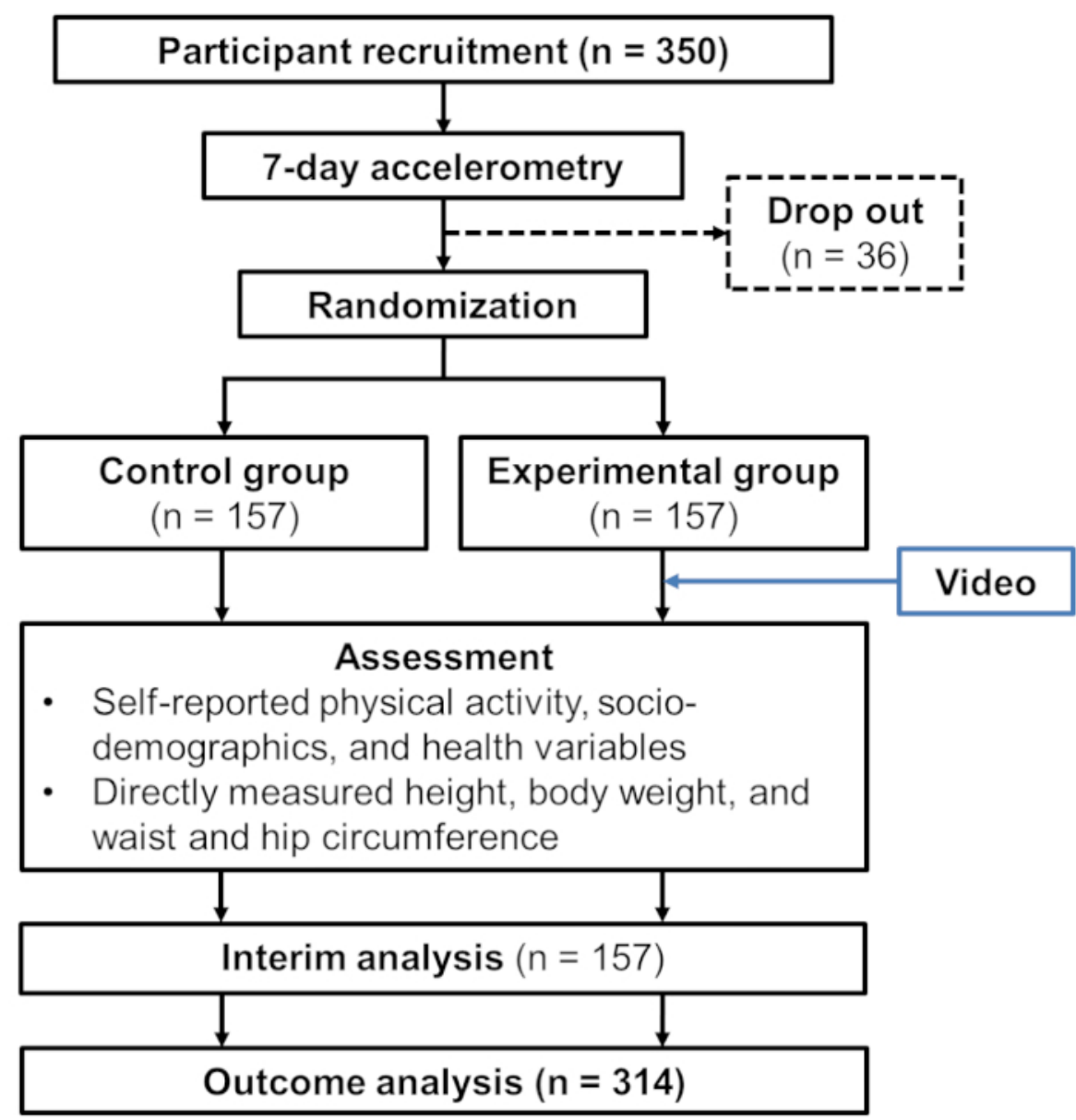

Figure 2: Schematic depiction of the calculated participation flow. $n=$ number of participants. All $\mathrm{n}$ refer to results of the power calculation. Please click here to view a larger version of this figure.

\section{Participant recruitment and preparation for data collection}

1. Choose a recruitment setting that permits enough time to hand out the accelerometer and to prepare it for data collection (e.g., in a shopping mall or at the workplace) in order to keep efforts of participants low and to increase adherence to the study.

1. Recruit participants who have the ability to walk independently (e.g., no permanent use of a wheelchair) and who are physically and cognitively capable of completing a self-report questionnaire. Be sure to recruit a similar number of male and female participants of all ages within the desired age range.

2. As an incentive for participation, point out that participants are going to receive a feedback letter on directly measured PA and sedentary time after completing the study. Use monetary incentives as desired.

3. Obtain written informed consent from each person prior to their participation.

2. For objective measurement, use a three-axial accelerometer to be worn on the right hip. Alternative devices can be used but should have the memory capacity for data collection on seven consecutive days. In order to most accurately capture daily PA, follow the instructions of the specific device used.

1. Have accelerometers prepared with elastic belts of various sizes and select one that fits the participant comfortably.

2. Give adequate information on how to handle the accelerometer according to instructions provided by the producer of the device. Instruct participants to start wearing the device on the next day. Ensure that participants wear the device during waking hours (i.e., every day after getting up until going to sleep).

3. Initialize the accelerometer on a computer using the appropriate software. Make sure to set up the wearing period correctly. Select a sampling rate of $30 \mathrm{~Hz}^{23}$. If applicable, choose to fill in participant specific information as desired (e.g., body weight or date of birth for reasons of participant identification).

4. Schedule each participant for an assessment session to obtain self-reported PA and somatometry. Ensure this session takes place one day after the last accelerometer wearing day. Hence, accelerometer and questionnaire data refer to the same period of time. If this is not possible for reasons of time, admit a maximum delay of two days.

5. Dismiss the participant with encouragement to engage in normal daily activities and make sure the participant remembers to return the accelerometer when appearing for the session.

NOTE: This study is conducted in Greifswald, a city in Western Pomerania, a rural area in northeastern Germany. Persons from the general population aged between 40 and 75 years are recruited proactively at a shopping mall. Accelerometer feedback letters 
and shopping vouchers in the amount of 10 euros are used as incentives. Participants are instructed to wear the device for seven consecutive days and to remove it for any water-based activities (e.g., showering or swimming).

\section{Participant assessment session}

NOTE: Conduct this session within three days after the last accelerometer wearing day.

1. Collect the accelerometer from the participant.

2. Set up a new participant in the tablet-computer survey and type in the individual study identification number of the participant.

3. Hand over the tablet computer to the participant to answer the self-administrative questionnaire.

4. When the participant has completed the questionnaire, collect the tablet computer and continue with measurement of somatometry.

1. Ask the participant to take off their shoes and to stand on calibrated scales for measurement of body weight. Type in the result into the tablet computer.

2. Ask the participant to stand up straight in front of a mirror with toes at a mark on the ground for measurement of body height. Type in the result into the tablet computer.

3. Ask the participant to remove upper layers of clothing for measurement of waist and hip circumference. Measure waist circumference midway between lowest rib and iliac crest. Measure hip circumference about two inches below iliac crest. Use the mirror to check accurate positioning of the tape. Type in the results into the tablet computer.

5. Thank and dismiss the participant.

\section{Download of accelerometer data for processing and creation of feedback letters}

1. Download the data from the device using the appropriate software.

1. Select to use data from the vertical axis and choose an epoch length of $10 \mathrm{~s}$.

2. Export the data to an appropriate program for further processing. According to the output metric used, choose cut points to determine non-wear time and to differentiate between PA intensity levels ${ }^{24,25}$.

1. Define non-wear time as at least $60 \mathrm{~min}$ of consecutive zero counts, allowing for $\leq 2 \mathrm{~min}$ of counts between 0 and $100^{24}$.

2. In an adult sample (ages 18 or older), classify values $<100$ counts per min as sedentary time, values between 100 and 2019 counts per min as light PA, values between 2020 and 5998 as moderate PA and values of 5999 or more counts per min as vigorous $\mathrm{PA}^{24}$.

2. Import all relevant variables into a computer program appropriate for creating a computerized feedback letter using an algorithm to automatically integrate the individual data into a general template. The letter may contain a number of graphs visualizing accelerometerbased PA outcomes as well as sedentary time as desired. Have each graph accompanied by a paragraph of three to five sentences explaining the content of the figures and providing respective health recommendations.

3. Deliver the feedback letter as soon as possible after the participant completed the study. NOTE: Accelerometer feedback letters in the present study include three graphs. The first graph visualizes daily steps across the wearing period. The second graph shows amounts of time spent sedentary and in light, moderate, and vigorous PA on each wearing day. The third graph depicts all observed 10-min-bouts of sedentary time between 6 and $10 \mathrm{pm}$ exemplified on a weekday and on a weekend day. Recommendations on PA are presented according to the PA guidelines of the World Health Organization for apparently healthy adults ${ }^{2}$. Recommendations on sedentary breaks are presented based on relevant studies ${ }^{26,27,28}$.

\section{Statistical analysis}

1. Calculate descriptive statistics for all variables.

2. Define a cut-off value for daily accelerometer wear time to avoid bias in accelerometer data.

3. Create a variable that presents the gap between the two measures. Calculate the variable as self-reported minus accelerometer-derived min of moderate-to-vigorous PA which results in a difference score (delta, $\Delta$ ). Use a two-sample t-test to determine the difference of deltas between experimental and control group.

4. Create a graph to visualize the results of the main analysis as desired.

\section{Representative Results}

The methods detailed above describe a randomized controlled trial to test whether a video demonstration of PA intensity levels reduces the gap between self-reported and accelerometer-based MVPA. An interim analysis $(n=157)$ was planned to evaluate whether the estimated sample size of 314 participants is sufficient to test our hypothesis. Up to this point, 142 participants completed the study protocol. Participants who were too old $(n=1)$ or who did not wear the accelerometer for $\geq 10$ hours per day on $\geq 6$ days $(n=10)$ were excluded from the analysis. Thus, data analysis was carried out using a sample of 131 participants to give an example of representative results among individuals from the general population aged between 40 and 75 years. 
Table 1 presents descriptive statistics of the analysis sample $(n=131)$. Of this sample, 68 participants $(52 \%)$ were randomized to the experimental group and 63 participants $(48 \%)$ were randomized to the control group. The experimental group received a video demonstration before completing the PA questionnaire, whereas the control group received PA assessment only. It was hypothesized that the video demonstration reduces the gap between self-reported and accelerometer-based PA. Preliminary results of interim analysis revealed a lower formal mean difference in the video group $(M=21.8, S D=108.9)$ compared to controls $(M=41.0, S D=117.4, t(129)=0.97, p=.166$, Figure 3 and Figure 4). The $p$-value lies between the significance $(p<0.010)$ and futility $(p>0.269)$ boundaries of the test simulations. Thus, the study may continue as planned until the total sample size is reached.

\begin{tabular}{|c|c|c|c|}
\hline & Total Sample & Control group & Video group \\
\hline $\mathrm{N}$ & 131 & $63(48 \%)$ & $68(52 \%)$ \\
\hline Sex, women & $85(65 \%)$ & $46(73 \%)$ & $39(57 \%)$ \\
\hline Age, years & $60.1 \pm 8.9$ & $58.1 \pm 9.6$ & $61.9 \pm 7.9$ \\
\hline $\begin{array}{l}\text { Current living together with a } \\
\text { partner, yes }\end{array}$ & $102(78 \%)$ & $51(81 \%)$ & $51(75 \%)$ \\
\hline \multicolumn{4}{|l|}{ School education } \\
\hline$<10$ years & $20(16 \%)$ & $12(19 \%)$ & $8(12 \%)$ \\
\hline 10 years & $64(50 \%)$ & $27(44 \%)$ & $37(56 \%)$ \\
\hline$>10$ years & $44(34 \%)$ & $23(37 \%)$ & $21(32 \%)$ \\
\hline \multicolumn{4}{|l|}{ Not specified $(n=3)$} \\
\hline \multicolumn{4}{|l|}{ Employment } \\
\hline Full-time or part-time & $55(42 \%)$ & $33(52 \%)$ & $22(32 \%)$ \\
\hline Irregularely & $23(18 \%)$ & $8(13 \%)$ & $15(22 \%)$ \\
\hline Not employed or retired & $53(40 \%)$ & $22(35 \%)$ & $31(46 \%)$ \\
\hline Current smoker, yes & $22(17 \%)$ & $12(19 \%)$ & $10(15 \%)$ \\
\hline \multicolumn{4}{|l|}{ Body mass index } \\
\hline$<25 \mathrm{~kg} / \mathrm{m}^{2}$ & $34(26 \%)$ & $23(37 \%)$ & $11(16 \%)$ \\
\hline$\geq 25 \mathrm{~kg} / \mathrm{m}^{2}$ and $<30 \mathrm{~kg} / \mathrm{m}^{2}$ & $55(42 \%)$ & $22(35 \%)$ & $33(49 \%)$ \\
\hline$\geq 30 \mathrm{~kg} / \mathrm{m}^{2}$ & $42(32)$ & $18(29 \%)$ & $24(35 \%)$ \\
\hline Self-reported general health & $2.8 \pm 0.7$ & $2.8 \pm 0.8$ & $2.8 \pm 0.6$ \\
\hline Accelerometer wear time, $\mathrm{min} /$ day & $883.0 \pm 82.8$ & $896.1 \pm 74.4$ & $870.8 \pm 88.7$ \\
\hline $\begin{array}{l}\text { Accelerometer-based MVPA, min/ } \\
\text { day }\end{array}$ & $45.2 \pm 27.7$ & $44.1 \pm 24.3$ & $46.2 \pm 30.7$ \\
\hline Self-reported MVPA, min/day & $77.2 \pm 117.2$ & $85.2 \pm 119.0$ & $68.0 \pm 115.8$ \\
\hline
\end{tabular}

Table 1: Sample characteristics of participants included in the preliminary interim analysis. $N=$ number of participants. $M V P A=$ moderate-to-vigorous physical activity. Data are presented as mean \pm standard deviation for continuous variables and as the number of participants (\%) for categorical variables. Body mass index was calculated from objectively measured height and body weight at the participant assessment session. Self-reported general health was measured on a 5-point scale from 1 "very good" to 5 "very bad". Self-reported and accelerometer-based MVPA as well as accelerometer wear time refer to average minutes per day across seven days. 


\section{Mean $\triangle$ of self-reported and accelerometer- based MVPA (min/day)}

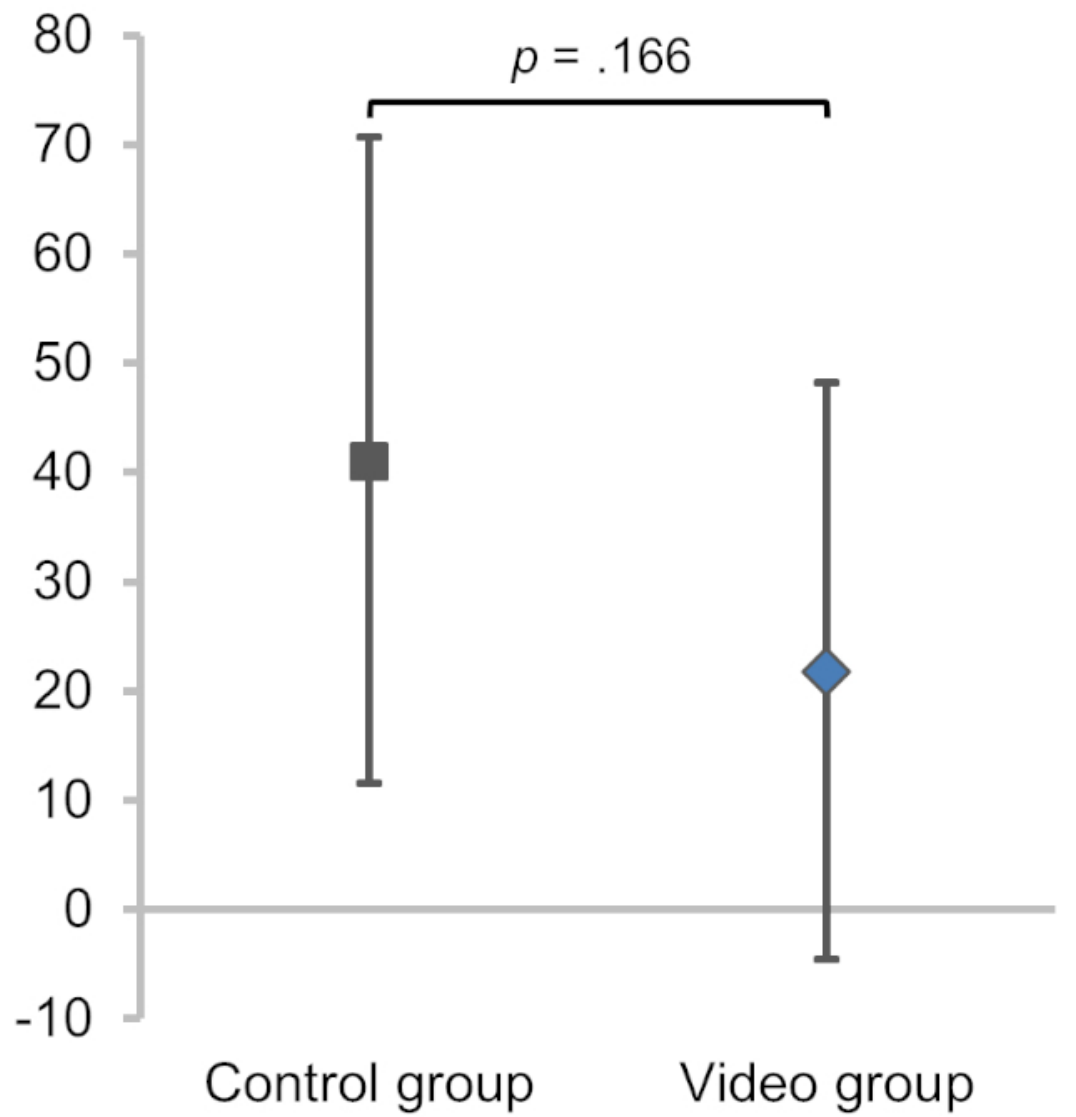

Figure 3: Mean difference between self-reported and accelerometer-based moderate-to-vigorous physical activity compared between study groups. $\triangle=$ delta. MVPA = moderate-to-vigorous physical activity. $\mathrm{min} /$ day $=$ minutes per day. The mean differences with according $95 \%$ confidence intervals of the control group (grey square) and the video group (blue diamond) are depicted. Mean differences were calculated as self-reported minus accelerometer-derived min of MVPA. The data refer to preliminary results of interim analysis $(n=131)$. Please click here to view a larger version of this figure.
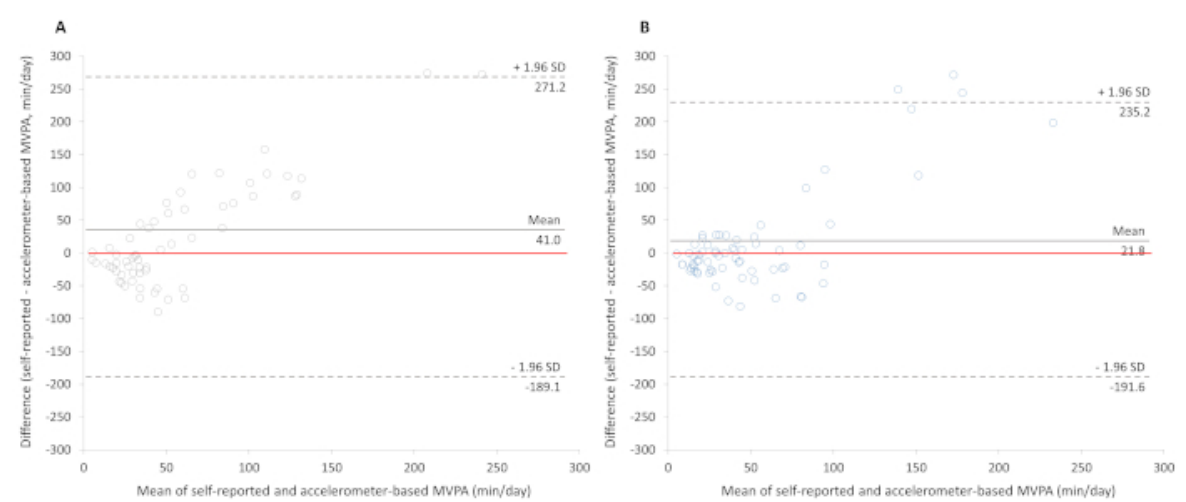

Figure 4: Bland Altman plots for visual depiction of the difference between self-reported and accelerometer-based moderate-tovigorous physical activity in the control group $(\mathrm{A})$ and in the video group (B). MVPA = moderate-to-vigorous physical activity. min/day = minutes per day. $S D=$ standard deviation. Differences were calculated as self-reported minus accelerometer-derived min of MVPA. A perfect agreement between the measures would be present if all dots lied on a horizontal line at the value 0 of the $y$-axis (red line). The data refer to preliminary results of interim analysis $(n=131)$. Please click here to view a larger version of this figure. 


\section{Discussion}

This report describes a methodology for testing the effect of a video demonstration on the gap between self-reported and accelerometer-based PA. If self-report assessment is preceded by a video demonstration of PA intensity levels, over-reporting of MVPA might be reduced. This protocol can be used to test the effect of any existing or self-produced information video on the gap between self-reported PA data derived from a computer-assisted assessment and directly measured PA.

The most important steps in the protocol include fundamental aspects of trial conduction that ensure the receipt of accurate data, such as correct accelerometer initialization and data download or making sure that the video may not be skipped by respondents. Further, there are more specific issues about the accelerometer wearing period and the daily wear time. First, the accelerometer wearing period and self-reported data should refer to the same time frame. To hand out accelerometers and agree on the date of the assessment session immediately after recruitment seems helpful to ensure participants' adherence to the scheduled appointment. Second, participants may not always comply with the instructions for accelerometer wearing. The device may be worn for less than seven days and/or only a few hours per day, whereas subsequent self-reports refer to the complete wearing period. Thus, over-reporting of MVPA may be bound to occur. Moreover, if wear time substantially differs between study groups, results may be compromised due to biased accelerometer-based MVPA data. Inspection of interim descriptive statistics may uncover insufficient amounts of wear time. For example, among the participants who completed the study protocol $(n=142)$, only 115 participants wore the device at least 10 hours on each of the seven days. There were three participants with a wear time of 0 minutes on one or more days. Excluding outliers seems necessary to ensure that the data are representative for an entire day as well as the total assessment period. Although most studies on correlations between accelerometry and PA questionnaire data request a wear time of $\geq 10$ hours per day on $\geq 4$ days per week ${ }^{29}$, investigations on the gap between measures may require more conservative cut-off values. Thus, we decided to exclude participants from the analysis who did not wear the accelerometer for $\geq 10$ hours per day on $\geq 6$ days.

Further modifications of the protocol may be appropriate. Preliminary results of descriptive statistics shown in Table 1 indicate an unbalanced proportion of men and women in our total sample and between study groups. If the video affects self-reports differentially in men and women, overall video effects may be biased. Thus, basic variables (e.g., sex and age) may need to be considered in the randomization algorithm. Moreover, the main analysis model may need to include sociodemographic and health related variables as potential confounders using a linear regression model instead of a t-test.

The methodology described here aims at reducing the gap between self-reported and accelerometer-derived PA by using a video to address comprehension of PA intensity levels. However, specific characteristics inherent to each measure remain to affect this gap. First, self-reported PA data is susceptible to recall bias ${ }^{30}$ and may be affected by social desirability bias ${ }^{31,32}$. Second, bias in accelerometer data particularly origins in different motivation to wear the device. Third, hip-worn accelerometers may lack the ability to accurately capture cycling and swimming ${ }^{13}$. Finally, accelerometers capture absolute amounts of movement whereas self-reports account for relative physical exertion ${ }^{33,34,35}$. Considering these factors, the visualization of intensity levels may present only one of many options to reduce the gap between self-reported and directly measured PA.

\section{Disclosures}

The authors have nothing to disclose.

\section{Acknowledgments}

This research was supported by the University Medicine Greifswald and the DZHK (German Centre for Cardiovascular Research; Grand No. D347000002). The authors wish to thank Christian Goeze, Stefanie Tobschall, and Clip Film- und Fernsehproduktion GmbH.

\section{References}

1. Arem, $\mathrm{H}$. et al. Leisure time physical activity and mortality: a detailed pooled analysis of the dose-response relationship. JAMA Internal Medicine. 175 (6), 959-967 (2015).

2. WHO - World Health Organization. Global recommendations on physical activity for health. http://www.who.int/dietphysicalactivity/ factsheet_adults/en/ (2018).

3. AHA - American Heart Association. American Heart Association recommendations for physical activity in adults., http://www.heart.org/en/ healthy-living/fitness/fitness-basics/aha-recs-for-physical-activity-in-adults (2018).

4. Craig, C. L. et al. International physical activity questionnaire: 12 -country reliability and validity. Medicine \& Science in Sports \& Exercise. 35 (8), 1381-1395 (2003).

5. Armstrong, T., Bull, F. Development of the World Health Organization Global Physical Activity Questionnaire (GPAQ). Journal of Public Health. 14 (2), 66-70 (2006).

6. Godin, G., Jobin, J., Bouillon, J. Assessment of leisure time exercise behavior by self-report: a concurrent validity study. Canadian Journal of Public Health. 77 (5), 359-362 (1986).

7. CDC/National Center for Health Statistics. National Health Interview Survey., https://www.cdc.gov/nchs/nhis/data-questionnairesdocumentation.htm (2018).

8. Friedenreich, C. M., Courneya, K. S., Bryant, H. E. The Lifetime Total Physical Activity Questionnaire: development and reliability. Medicine \& Science in Sports \& Exercise. 30, 266-274 (1998).

9. Finger, J. D. et al. How well do physical activity questions perform? A European cognitive testing study. Archives of Public Health. 73 (57) (2015). 
10. Baumann, S. et al. Pitfalls in accelerometer-based measurement of physical activity: the presence of reactivity in an adult population. Scandinavian Journal of Medicine \& Science in Sports. 28 (3), 1056-1063 (2018).

11. Clemes, S. A., Deans, N. K. Presence and duration of reactivity to pedometers in adults. Medicine \& Science in Sports \& Exercise. 44 (6), 1097-1101 (2012).

12. Weymar, F. et al. Characteristics associated with non-participation in 7-day accelerometry. Preventive Medicine Reports. 2, 413-418 (2015).

13. Young, D. R. et al. Sedentary behavior and cardiovascular morbidity and mortality: a science advisory from the American Heart Association. Circulation. 134 (13), e262-e279 (2016).

14. Cerin, E. et al. Correlates of agreement between accelerometry and self-reported physical activity. Medicine \& Science in Sports \& Exercise. 48 (6), 1075-1084 (2016).

15. Dyrstad, S. M., Hansen, B. H., Holme, I. M., Anderssen, S. A. Comparison of self-reported versus accelerometer-measured physical activity. Medicine \& Science in Sports \& Exercise. 46 (1), 99-106 (2014).

16. Lee, P. H., Macfarlane, D. J., Lam, T., Stewart, S. M. Validity of the international physical activity questionnaire short form (IPAQ-SF): A systematic review. International Journal of Behavioral Nutrition and Physical Activity. 8 (115) (2011).

17. Balachandran, A., Verduin, C. N., Potiaumpai, M., Ni, M., Signorile, J. F. Validity and reliability of a video questionnaire to assess physical function in older adults. Experimental Gerontology. 81, 76-82 (2016).

18. Marsh, A. P. et al. Assessing walking activity in older adults: development and validation of a novel computer-animated assessment tool. The Journals of Gerontology. Series A, Biological Sciences and Medical Sciences. 70 (12), 1555-1561 (2015).

19. Marsh, A. P. et al. The Virtual Short Physical Performance Battery. The Journals of Gerontology. Series A, Biological Sciences and Medical Sciences. 70 (10), 1233-1241 (2015).

20. Centers for Disease Control and Prevention (CDC). Physical Activity Guidelines - What Counts As Aerobic?., https://www.youtube.com/ watch?v=GEvJImpZCoM (2012).

21. Hagströmer, M. Downloadable questionnaires., https://www.sites.google.com/site/theipaq/questionnaire_links (2016).

22. Ainsworth, B. E. et al. Compendium of physical activities: an update of activity codes and MET intensities. Medicine \& Science in Sports \& Exercise. 32 (9 Suppl), 498-504 (2000).

23. Migueles, J. H. et al. Accelerometer data collection and processing criteria to assess physical activity and other outcomes: a systematic review and practical considerations. Sports Medicine. 47 1821-1845 (2017).

24. Troiano, R. et al. Physical activity in the United States measured by accelerometer. Medicine \& Science in Sports \& Exercise. 40, 181-188 (2008).

25. Freedson, P., Melanson, E., Sirard, J. Calibration of the computer science and applications, inc. accelerometer. Medicine \& Science in Sports \& Exercise. 30, 777-781 (1998).

26. Duvivier, B. M. F. M. et al. Benefits of substituting sitting with standing and walking in free-living conditions for cardiometabolic risk markers, cognition and mood in overweight adults. Frontiers in Physiology. 8 (2017).

27. Benatti, F. B., Ried-Larsen, M. The effects of breaking up prolonged sitting time: a review of experimental studies. Medicine \& Science in Sports \& Exercise. 47 (10), 2053-2061 (2015).

28. Chastin, S. F. M., Egerton, T., Leask, C., Stamatakis, E. Meta-analysis of the relationship between breaks in sedentary behavior and cardiometabolic health. Obesity. 23, 1800-1810 (2015).

29. Skender, S. et al. Accelerometry and physical activity questionnaires - a systematic review. BMC Public Health. 16, (515) (2016).

30. Herbolsheimer, F., Riepe, M. W., Peter, R. Cognitive function and the agreement between self-reported and accelerometer-accessed physical activity. BMC Geriatrics. 18 (56) (2018).

31. Motl, R. W., McAuley, E., DiStefano, C. Is social desirability associated with self-reported physical activity? Preventive Medicine. 40 (6), 735-739 (2005).

32. Adams, S. A. et al. The effect of social desirability and social approval on self-reports of physical activity. American Journal of Epidemiology. 161 (4), 389-398 (2005)

33. Kelly, P., Fitzsimons, C., Baker, G. Should we reframe how we think about physical activity and sedentary behaviour measurement? Validity and reliability reconsidered. International Journal of Behavioral Nutrition and Physical Activity. 13 (32) (2016).

34. Troiano, R. P., McClain, J. J., Brychta, R. J., Chen, K. Y. Evolution of accelerometer methods for physical activity research. British Journal of Sports Medicine. 48 (13), 1019-1023 (2014).

35. Shook, R. P. et al. Subjective estimation of physical activity using the International Physical Activity Questionnaire varies by fitness level. Journal of Physical Activity \& Health. 13 79-86 (2016). 